\section{Occurrence of harmful algal species and shellfish toxicity in Sardinia (Italy)}

\author{
Anna Maria Bazzoni," \\ Alessandro Graziano Mudadu, \\ Giuseppa Lorenzoni, ${ }^{1}$ Igor Arras, ${ }^{1}$ \\ Antonella Lugliè, ${ }^{2}$ Barbara Vivaldi, ${ }^{3}$ \\ Valentina Cicotelli, ${ }^{3}$ Giovanna Sanna, \\ Giuseppe Tedde, ${ }^{1}$ Salvatore Ledda, ${ }^{1}$ \\ Enrico Alesso, ${ }^{3}$ Edoardo Marongiu, ${ }^{1}$ \\ Sebastiano Virgilio' \\ ${ }^{1}$ Institute for Experimental Veterinary \\ Medicine of Sardinia, Sassari; ${ }^{2}$ University \\ of Sassari, Sassari; ${ }^{3}$ Institute for \\ Experimental Veterinary Medicine of \\ Piedmont, Liguria and Valle D'Aosta, \\ Genoa, Italy
}

\section{Abstract}

Sardinia (Italy, north-western Mediterranean) is a commercially important producer of edible bivalve molluscs. Since the early 2000 s, it was subjected to recurring cases of mussel farm closures due to toxic algal poison. Here, we present the studies on toxin concentrations and the associated potentially toxic phytoplankton distribution and abundances carried out by a regular monitoring programme in Sardinian shellfish areas, from January to May 2015. Diarrheic shellfish poisoning (DSP) toxins were detected in several bivalve molluscs samples, while paralytic shellfish poisoning (PSP) and paralytic shellfish poisoning toxins were present just once, without exceeding the legal limits. Potentially toxic algal species have been constantly present. Pseudo-nitzschia species were present during the entire study often with high abundances, while Dinophysis species reached high densities sporadically. Among PSP phytoplankton, only Alexandrium minutum Halim was found. The data obtained in this study showed an increase in the DSP toxicity in mussels in Sardinia. No clear relation between the occurrence of toxins in shellfish and the presence of potentially toxic algal species was found, although a slight correlation between DSP toxins and Dinophysis species could be supported.

\section{Introduction}

The last two decades have been marked by an expansion of potentially toxic harmful algal species (HAS) throughout the world (Hallegraeff, 2010). The uptake of algal toxins by suspension feeding bivalve molluscs is the main process through which different phycotoxins, with different impacts [such as amnesic shellfish poisoning (ASP); diarrheic shellfish poisoning (DSP); paralytic shellfish poisoning (PSP)], move from the marine food chain to human beings, involving intoxications. For this reason, the potentially toxic HAS represent a significant threat to human health and aquaculture resources, and the intoxication after the contaminated bivalve molluscs ingestion is a worldwide problem (Taleb et al., 2003). Nowadays, shellfish poisoning linked to potentially toxic HAS is a well-known phenomenon (Hallegraeff, 2010) and we are more aware than in the past regarding the size and complexity of the problem.

Shellfish farming is widespread along the Sardinian coastline. To this purpose, this region has relevant economic and social interests in this commercial field (Mazzette et al., 2010). Recurring occurrence of potentially toxic HAS in shellfish producing areas implies contamination and a consequent human poisoning risk brought by contaminated seafood consumption. An intense monitoring network was set up in every shellfish farm, in order to protect and defend the consumer and aquaculture operators from economic loss. Since 1988, in Sardinia there is an operating monitoring programme coordinated by the Prevention Department of Health of the Autonomous Region of Sardinia (Lorenzoni et al., 2013; Bazzoni et al., 2015). Previous studies (Sannio et al., 1997; Bazzoni et al., 2015) showed the presence and extensive distribution of potentially toxic HAS along Sardinian coast, with different trends and impacts. DSP and PSP toxins have become widespread, while ASP toxin values never exceeded regulatory limits in mussels. The aim of this paper is to show the regional monitoring results about the potentially toxic HAS presence (Dinophysis spp., Prorocentrum spp., Pseudo-nitzschia spp., Alexandrium spp.) and the toxin content [Okadaica group and total derivates,; domoic acid (DA); saxitoxin group (STX)] in mussels. Here we specifically report toxins concentration data found in 2015 when the levels exceeded the permitted limits by current regulation (EC Reg. n. 853/2004; European Commission, 2004), as well as the potentially toxic phytoplankton distribution and abundances associated.

\section{Materials and Methods}

\section{Study area and sampling}

The study was conducted from January to May 2015 along Sardinian coastline (Italy, NW Mediterranean Sea), in mussel cultivating areas: Cagliari area, including Santa Gilla
Correspondence: Anna Maria Bazzoni, Department of Food Hygiene, Institute for Experimental Veterinary Medicine of Sardinia, via Duca degli Abruzzi 8, 07100 Sassari, Italy. Tel: +39.0792892348 - Fax: +39.0792892324.

E-mail: bazzoni.annamaria@tiscali.it

Key words: Biotoxins; Potentially toxic microalgae; Shellfish farms; Mediterranean.

Conflict of interest: the authors declare no potential conflict of interest.

Received for publication: 16 June 2016.

Revision received: 29 August 2016.

Accepted for publication: 29 August 2016.

This work is licensed under a Creative Commons Attribution-NonCommercial 4.0 International License (CC BY-NC 4.0).

(C) Copyright A.M. Bazzoni et al., 2016 Licensee PAGEPress, Italy

Italian Journal of Food Safety 2016; 5:6095 doi:10.4081/ijfs.2016.6095

(SGL; stations 1,2,3), San Giovanni (SGN; stations 1, 2, 3, 4), Feraxi (FER; stations 1, 2,8), Colostrai (COL; station 1); Olbia area, including Porto Pozzo (PPZ), Gulf of Olbia (GOL), San Teodoro (STD); Oristano area, including Cabras (CAB), Torregrande (TGR), S'Ena Arrubia (SEA), Torrevecchia (TVC), Capo San Marco (CSM; station 1), Corru Mannu (CMN), III Peschiera (PES); East area with Tortolì (TOR; stations 1,2,3,4) and Orosei (OR0; stations 1,4) (Figure 1). On the same dates, water samples and mussels samples were collected in order to assess, respectively, the potentially toxic HAS presence and the ASP, DSP and PSP toxins detection. According to the Sardinian Regional Monitoring Programme (Regione Sardegna, 2014), the samples were collected weekly (Cagliari area) or bi-weekly (Oristano, Olbia and East areas). The sampling frequency increased when mussel toxicity exceeded the legal limits (EC Reg. n. 853/2004; European Commission, 2004), above control levels every two days. The progressive sample sequencing was labeled monthly using Roman numerals.

\section{Potentially toxic harmful algal species}

A total of 570 water samples were examined. The samples $(1 \mathrm{~L})$ were taken in clean PE bottles at a depth of $0.5 \mathrm{~m}$ from the water surface and immediately fixed with Lugol's iodine solution or with $4 \%$ solution of formaldehyde in order to preserve them for further analysis within $24 \mathrm{~h}$. The analyses were performed using settling chambers (from 5 to $25 \mathrm{~cm}^{3}$ depending on phytoplankton cell densities) under an inverted microscope (Olympus IX 73; 
Olympus, Center Valley, PA, USA). According to Utermöhl's method (1958), all specimens of potentially toxic HAS, referring to IOC Taxonomic Reference List of Toxic Plankton Algae (http://www.marinespecies.org/hab/), were counted on the whole settling chamber surface at a $\times 200$ magnification.

\section{Toxins}

A total of 559 shellfish samples were examined for toxins analysis. In accordance with AOAC 959.08 (AOAC, 2005), PSP toxins were extracted from shellfish tissue. An aliquot of $100 \mathrm{~g}$ from each sample was homogenized (SUNBEAM mod 4153-50) and extracted with $100 \mathrm{~mL}$ of $\mathrm{HCl} 0.1 \mathrm{~N}$. pH was adjusted approximately around 3 and the mixture was boiled for 5 minutes. After cooling to room temperature, the $\mathrm{pH}$ was again adjusted by addition of either $\mathrm{HCl} 0.5 \mathrm{~N}$ or $\mathrm{NaOH} 0.1 \mathrm{~N}$. Water was added up to a total volume of $200 \mathrm{~mL}$. After 5 minutes centrifugation at 3000 r.p.m., $1 \mathrm{~mL}$ of translucent supernatant of mixture was injected intraperitoneally into 3 mice. The symptoms, in terms of mouse behavior after injection, were observed, and lethal time was recorded. The test was considered positive when the mice (Swiss mice with 19-21 g body weight) died within a specific time period and the death time was used to quantify the level of toxin present. According to the official protocol (AESAN, 2015), the determination of DSP toxins (okadaic acid, OA; dinophysistoxins, DTXs; pectenotoxins, PTXs; yessotoxins, YTXs; azaspiracids, AZAs) were carried out using the low chromatography tandem mass spectrometry approach (LC-MS/MS). It is governed by EC Regulation 15/2011 (European Commission, 2011) and was tested on a secondary validation compared to this rule, for all groups except for the PTXs. An aliquot ( $2 \mathrm{~g}$ ) of muscle tissue was homogenized with $9 \mathrm{~mL}$ of methanol. After shaking, the extract was cen-

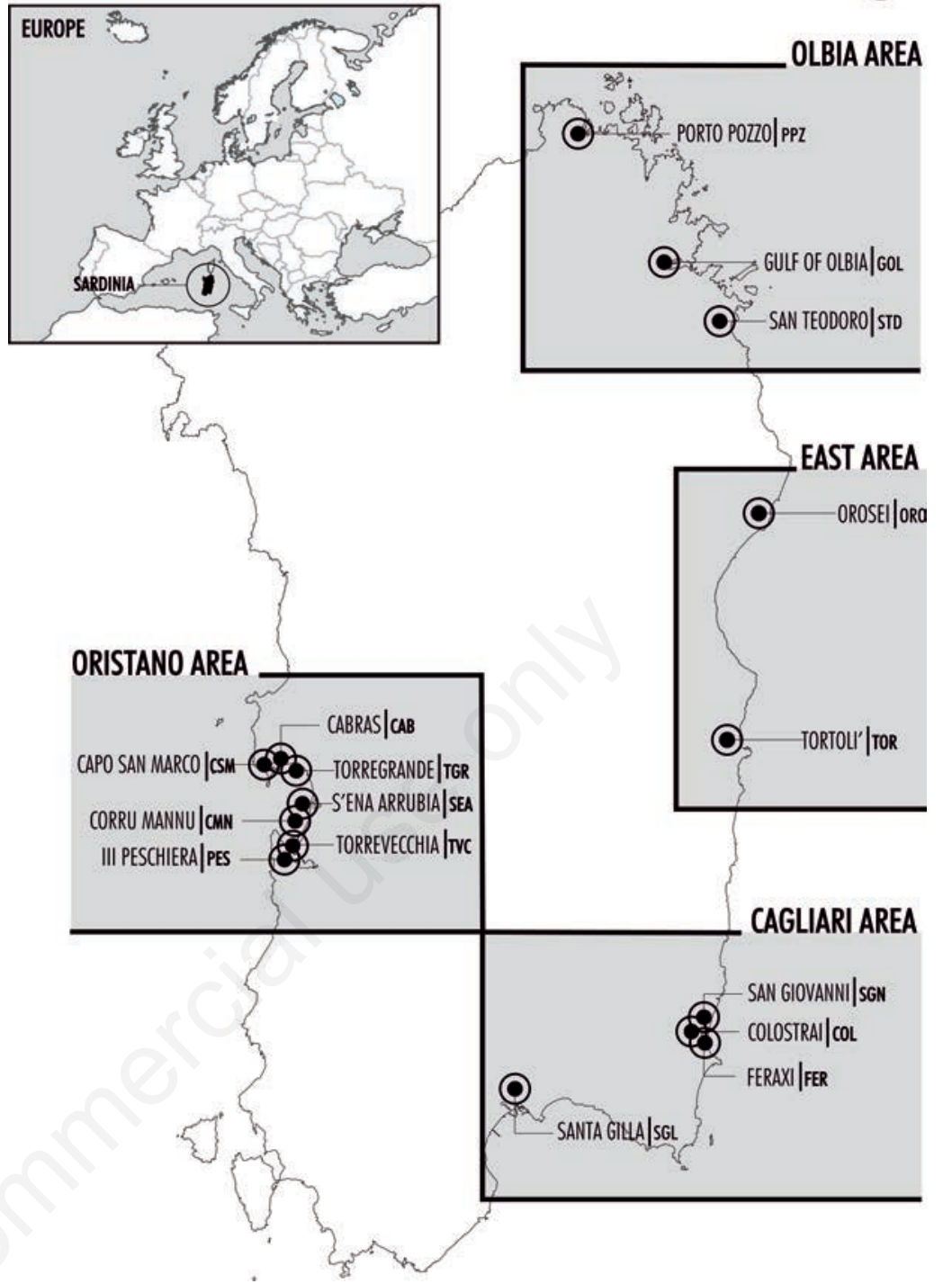

Figure 1. Study area and location of sampling sites.

Table 1. Tandem mass spectrometry conditions for the selected reaction monitoring acquisition of marine lipophilic toxins.

\begin{tabular}{|c|c|c|c|c|c|}
\hline Toxin & ESI mode & Precursor ion $(\mathrm{m} / \mathrm{z})$ & Product ion $(\mathrm{m} / \mathrm{z})$ & S-lens (V) & Collision energy (V) \\
\hline $\mathrm{OA}$ & $\mathrm{ESI}^{+}$ & 827.2 & $\begin{array}{l}723.4 \\
809.5\end{array}$ & 276 & $\begin{array}{l}45 \\
42\end{array}$ \\
\hline DTX1 & $\mathrm{ESI}^{+}$ & 841.4 & $\begin{array}{l}737.4 \\
823.5\end{array}$ & 251 & $\begin{array}{l}46 \\
39 \\
\end{array}$ \\
\hline DTX2 & $\mathrm{ESI}^{+}$ & 827.2 & $\begin{array}{l}723.4 \\
809.5\end{array}$ & 276 & $\begin{array}{l}45 \\
42\end{array}$ \\
\hline YTX & $\mathrm{ESI}^{-}$ & 1141.3 & $\begin{array}{c}1062.0 \\
855.1\end{array}$ & 248 & $\begin{array}{l}46 \\
67\end{array}$ \\
\hline Homo-YTX & $\mathrm{ESI}^{-}$ & 1155.2 & $\begin{array}{c}869.7 \\
1076.0\end{array}$ & 263 & $\begin{array}{l}78 \\
28\end{array}$ \\
\hline AZAl & $\mathrm{ESI}^{+}$ & 842.3 & $\begin{array}{l}824.4 \\
806.4\end{array}$ & 223 & $\begin{array}{l}30 \\
35\end{array}$ \\
\hline AZA2 & $\mathrm{ESI}^{+}$ & 856.3 & $\begin{array}{l}838.6 \\
654.3\end{array}$ & 210 & $\begin{array}{l}29 \\
44\end{array}$ \\
\hline AZA3 & $\mathrm{ESI}^{+}$ & 828.2 & $\begin{array}{l}810.5 \\
792.3\end{array}$ & 218 & $\begin{array}{l}29 \\
40\end{array}$ \\
\hline
\end{tabular}

ESI, electrospray ionization; OA, okadaic acid; DTX1, dynophysistoxin 1; DTX2, dynophysistoxin 2; YTX, azaspiracid; AZA1, azaspiracid 1; AZA2, azaspiracid 2; AZA3, azaspiracid 3. 
trifuged and the supernatant was transferred into a flask. The extraction was repeated a second time. The extracts were combined and brought to a final volume of $20 \mathrm{~mL}$ with methanol. The determination of OA, AZA and YTX was performed after filtering an aliquot of the methanolic extract through a $0.45 \mu \mathrm{m}$ syringe filter and injecting it onto LC-MS/MS. A second aliquot was subjected to basic hydrolysis for the determination of the total content of OA/DTXs. $125 \mu \mathrm{L}$ of $\mathrm{NaOH} 2.5 \mathrm{M}$ was added to 1 $\mathrm{mL}$ of extract and the mix was heated at $76^{\circ} \mathrm{C}$ for 40 minutes. Then, after cooling to room temperature, it was neutralized with $125 \mu \mathrm{L}$ of $\mathrm{HCl} 2.5$ $\mathrm{M}$ and homogenized in vortex for 0.5 minutes. This extract was filtered and injected onto LCMS/MS. The analysis was performed using an HPLC Accela 1250 pump (Thermo Scientific, Waltham, MA, USA). Chromatographic separation was obtained by a reversed-phase Kinetex XB-C18 (100x2.1 mm ID, $2.6 \mu \mathrm{m}$; Phenomenex, Torrance, CA, USA). The eluents were $6.7 \mathrm{mM}$ ammonia aqueous solution (eluent A) and 6.7 $\mathrm{mM}$ ammonia solution in acetonitrile: water $90: 10 \mathrm{v} / \mathrm{v}$ (eluent B), at flow-rate of $450 \mu \mathrm{L} / \mathrm{min}$ utes. The elution gradient consisted of the following steps: from 0 to 1 minute, A at 95\%; from 13 to 13.5 minutes, A at $10 \%$; from 14 to $18 \mathrm{~min}$, $\mathrm{A} \%$ at $95 \%$. The injection volume was $5 \mu \mathrm{L}$. Mass spectral analysis was performed on a TSQ Vantage triple quadrupole mass spectrometer (Thermo Scientific), equipped with a heated ion spray interface (HESI). Detection and quantification of molecules (AESAN, 2015) were performed by selected reaction monitoring (SRM) (Table 1).

\section{Results}

Only Alexandrium minutum Halim was found among PSP phytoplankton, and just once Alexandrium sp. in January. All findings were detected in Oristano area (mainly in March), whilst in Cagliari, Olbia and East areas no findings were reported. Alexandrium abundance was always low, below $1000 \mathrm{cell} / \mathrm{L}$. The maximum abundance value (in CSM in the II sampling of March) was linked only STXs presence (503 $\mu \mathrm{g}$ eq STX/Kg e.p.) in shellfish (Figure 2). A number of Dinophysis (D. acuminata Claparède \& Lachmann, D. sacculus Stein, $D$. rotundata Claparède \& Lachmann, Dinophysis sp.) and Prorocentrum mexicanum OsorioTafall were usually found among DSP phytoplankton. They sporadically reached high densities, especially in TOR, where $D$. acuminata overcome $1 \times 10^{6}$ cell/L (January), and in ORO (Table 2). 23 bivalve shellfish samples (about $4 \%$ of total) were non-compliant for the DSP toxins presence over legal limit $(160 \mu \mathrm{g} 0 \mathrm{~A}$ eq/kg e.p.): 14 in a total of 246 (6\%) from Cagliari area, 2 in a total of 4 (50\%) from ORO and in 7 in a total of 94 (7\%) from TOR. The highest values of these toxins were found in February and March. In February there was the highest number of positive events (13), almost all recorded in Cagliari area (3 in SGL, 5 in FER, 1 in COL and 1 in SGN) and in part in TOR (3). The maximum toxin concentration (1480 $\mu \mathrm{g}$ OA eq/kg e.p.) was reported during the first sampling in SGL, in correspondence of which DSP phytoplankton was absent (Table 2, Figure 3). Positive samples were recorded in March as well in several shellfish farms. In COL there was a concentration of $470.4 \mu \mathrm{g} \mathrm{OA}$ eq/kg e.p. and absence of potentially toxic HAS (Table 2). As regard FER and TOR, the toxin values decreased but they remained still high, and while no evidence of DSP potentially causative species was found in FER, D. acuminata and $D$. sacculus were present in TOR and the sum of both reached a peak of about 20x103 cell/L in the station 3 (Table 2). In April positive samples were found in ORO (184 and $205 \mu \mathrm{g} \mathrm{OA} \mathrm{eq/kg} \mathrm{e.p.} \mathrm{in} \mathrm{the} \mathrm{two} \mathrm{sampling} \mathrm{sta-}$ tions) and TOR (210.1 $\mu$ g OA eq/kg e.p.) where D. acuminata showed elevated densities (about $2 \times 10^{3} \mathrm{cell} / \mathrm{L}$ in 0 R0 and $>9 \times 10^{3} \mathrm{cell} / \mathrm{L}$ in TOR). In May there was no positivity, even if DSP phytoplankton were present, especially in ORO and TOR (Table 2). ASP phytoplankton (Pseudo-nitzschia species) was found in almost $60 \%$ of samples, reaching the highest concentration in May in GOL $\left(>1 \times 10^{6}\right.$ cell/L).

Table 2. Okadaic acid group and total derivates quantification ( $\mu$ g eq OA/kg p.e.) in shellfish collected in Sardinia from January to May 2015 and corresponding potentially toxic harmful algal species densities (cell/L) in farm areas during positive events.

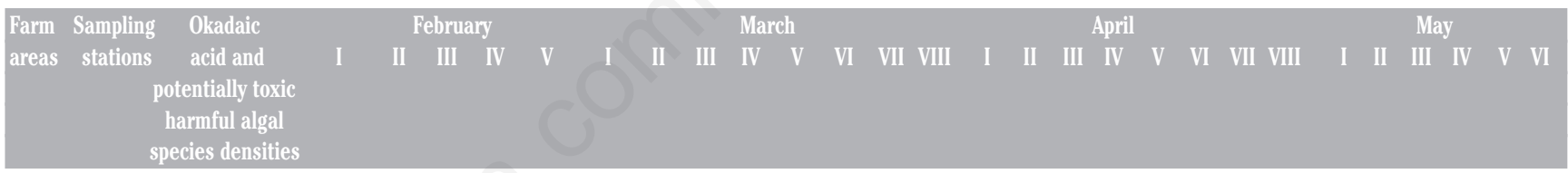

\begin{tabular}{|c|c|c|c|c|c|c|c|c|c|c|c|c|c|c|c|c|c|c|c|c|c|c|c|c|c|c|c|c|}
\hline COL & & $\begin{array}{c}\text { HAS DSP } \\
\text { DSP TOXINS }\end{array}$ & $\begin{array}{c}0 \\
\text { n.d. }\end{array}$ & $\begin{array}{c}0 \\
\text { n.d. }\end{array}$ & $\begin{array}{c}0 \\
341.3\end{array}$ & $\begin{array}{l}\text { n.s. } \\
\text { n.s. }\end{array}$ & $\begin{array}{l}\text { n.S. } \\
\text { n.S. }\end{array}$ & $\begin{array}{c}0 \\
\text { n.d. }\end{array}$ & $\begin{array}{c}0 \\
470.4\end{array}$ & $\begin{array}{c}0 \\
\text { n.d. }\end{array}$ & $\begin{array}{c}0 \\
\text { n.d. }\end{array}$ & $\begin{array}{c}0 \\
\text { n.d. }\end{array}$ & $\begin{array}{c}0 \\
\text { n.d. }\end{array}$ & $\begin{array}{c}0 \\
\text { n.d. }\end{array}$ & $\begin{array}{c}0 \\
\text { n.d. }\end{array}$ & $\begin{array}{c}0 \\
\text { n.d. }\end{array}$ & $\begin{array}{c}0 \\
\text { n.d. }\end{array}$ & $\begin{array}{c}0 \\
\text { n.d. }\end{array}$ & $\begin{array}{c}0 \\
\text { n.d. }\end{array}$ & $\begin{array}{c}0 \\
\text { n.d. }\end{array}$ & $\begin{array}{l}\text { n.s. } \\
\text { n.s. }\end{array}$ & $\begin{array}{l}\text { n.s. } \\
\text { n.s. }\end{array}$ & $\begin{array}{l}\text { n.s. } \\
\text { n.s. }\end{array}$ & $\begin{array}{cc}0 & 0 \\
\text { n.d. } & \text { n.d. }\end{array}$ & $\begin{array}{c}0 \\
\text { n.d. }\end{array}$ & $\begin{array}{c}0 \\
\text { n.d. }\end{array}$ & $\begin{array}{l}\text { n.s. } \\
\text { n.s. }\end{array}$ & $\begin{array}{l}\text { n.s. } \\
\text { n.s. }\end{array}$ \\
\hline FFR & 1 & $\begin{array}{c}\text { HAS DSP } \\
\text { DSP TOXINS }\end{array}$ & $\begin{array}{c}0 \\
1269\end{array}$ & $\begin{array}{c}0 \\
263.1\end{array}$ & $\begin{array}{c}0 \\
516.2\end{array}$ & $\begin{array}{c}0 \\
738.9\end{array}$ & $\begin{array}{c}0 \\
317\end{array}$ & $\begin{array}{r}40 \\
\text { n.d. }\end{array}$ & $\begin{array}{r}60 \\
\text { n.d. }\end{array}$ & $\begin{array}{c}0 \\
424.5\end{array}$ & $\begin{array}{c}0 \\
\text { n.d. }\end{array}$ & $\begin{array}{c}0 \\
383.1\end{array}$ & $\begin{array}{c}0 \\
257.2\end{array}$ & $\begin{array}{c}0 \\
\text { n.d. }\end{array}$ & $\begin{array}{c}0 \\
\text { n.d. }\end{array}$ & $\begin{array}{c}40 \\
133.6^{*}\end{array}$ & $\begin{array}{c}0 \\
\text { n.d. }\end{array}$ & $\begin{array}{c}40 \\
95.8^{*}\end{array}$ & $\begin{array}{c}0 \\
60.5^{*}\end{array}$ & $\begin{array}{c}0 \\
114.4\end{array}$ & $\begin{array}{c}0 \\
* \text { n.d. }\end{array}$ & $\begin{array}{c}0 \\
\text { n.d. }\end{array}$ & $\begin{array}{l}160 \\
\text { n.d. }\end{array}$ & $\begin{array}{cc}0 & 0 \\
\text { n.d. } & \text { n.d. }\end{array}$ & $\begin{array}{l}240 \\
\text { n.d. }\end{array}$ & $\begin{array}{l}1700 \\
\text { n.d. }\end{array}$ & & \\
\hline & 4 & $\begin{array}{c}\text { HAS DSP } \\
\text { DSP TOXINS }\end{array}$ & $\begin{array}{c}0 \\
\text { n.d. }\end{array}$ & $\begin{array}{c}0 \\
618.9\end{array}$ & $\begin{array}{l}\text { n.s. } \\
\text { n.s. }\end{array}$ & $\begin{array}{l}\text { n.s. } \\
\text { n.s. }\end{array}$ & & $\begin{array}{c}0 \\
\text { n.d. }\end{array}$ & $\begin{array}{c}0 \\
\text { n.d. }\end{array}$ & $\begin{array}{l}40 \\
\text { n.d. }\end{array}$ & $\begin{array}{c}0 \\
\text { n.d. }\end{array}$ & $\begin{array}{c}0 \\
\text { n.d. }\end{array}$ & $\begin{array}{c}0 \\
\text { n.d. }\end{array}$ & $\begin{array}{c}0 \\
\text { n.d. }\end{array}$ & $\begin{array}{l}\text { n.s. } \\
\text { n.s. }\end{array}$ & $\begin{array}{c}0 \\
\text { n.r. }\end{array}$ & $\begin{array}{c}0 \\
\text { n.r. }\end{array}$ & $\begin{array}{c}0 \\
\text { n.r. }\end{array}$ & $\begin{array}{c}0 \\
\text { n.r. }\end{array}$ & $\begin{array}{c}0 \\
\text { n.r. }\end{array}$ & $\begin{array}{c}0 \\
\text { n.r. }\end{array}$ & $\begin{array}{l}\text { n..s. } \\
\text { n..s. }\end{array}$ & $\begin{array}{l}\text { n.s. } \\
\text { n.s. }\end{array}$ & $\begin{array}{cc}0 & 0 \\
\text { n.r. } & \text { n.r. }\end{array}$ & $\begin{array}{c}0 \\
\text { n.r. }\end{array}$ & $\begin{array}{c}0 \\
\text { n.r. }\end{array}$ & & . n.s. \\
\hline SSI & 3 & $\begin{array}{c}\text { HAS DSP } \\
\text { DSP TOXINS } \\
\text { HAS DSP } \\
\text { DSP TOXINS }\end{array}$ & $\begin{array}{c}0 \\
1480 \\
0 \\
\text { n.d. }\end{array}$ & $\begin{array}{c}0 \\
\text { n.d. } \\
0 \\
\text { n.d. }\end{array}$ & $\begin{array}{c}0 \\
704.8 \\
0 \\
498.9\end{array}$ & $\begin{array}{c}0 \\
\text { n.d. } \\
0 \\
\text { n.d. }\end{array}$ & $\begin{array}{l}\text { n.s. } \\
\text { n.s. } \\
\text { n.d. } \\
\text { n.d. }\end{array}$ & $\begin{array}{c}0 \\
\text { n.d. } \\
0 \\
\text { n.d. }\end{array}$ & $\begin{array}{c}0 \\
\text { n.d. } \\
0 \\
\text { n.d. }\end{array}$ & $\begin{array}{c}0 \\
\text { n.d. } \\
300 \\
\text { n.d. }\end{array}$ & $\begin{array}{c}0 \\
\text { n.d. } \\
0 \\
\text { n.d. }\end{array}$ & $\begin{array}{c}0 \\
\text { n.d. } \\
0 \\
\text { n.d. }\end{array}$ & $\begin{array}{l}\text { n.s. } \\
\text { n.s. } \\
\text { n.s. } \\
\text { n.s. }\end{array}$ & $\begin{array}{l}\text { n.s. } \\
\text { n.s. } \\
\text { n.s. } \\
\text { n.s. }\end{array}$ & $\begin{array}{l}\text { n.s. } \\
\text { n.s. } \\
\text { n.s. } \\
\text { n.s. }\end{array}$ & $\begin{array}{c}0 \\
\text { n.d. } \\
0 \\
\text { n.d. }\end{array}$ & $\begin{array}{c}0 \\
\text { n.d. } \\
0 \\
\text { n.d. }\end{array}$ & $\begin{array}{c}0 \\
\text { n.d. } \\
0 \\
\text { n.d. }\end{array}$ & $\begin{array}{r}120 \\
\text { n.d. } \\
40 \\
\text { n.d. }\end{array}$ & $\begin{array}{c}0 \\
\text { n.d. } \\
0 \\
\text { n.d. }\end{array}$ & $\begin{array}{l}\text { n.s. } \\
\text { n.s. } \\
\text { n.s. } \\
\text { n.s. }\end{array}$ & $\begin{array}{l}\text { n.s. } \\
\text { n.s. } \\
\text { n.s. } \\
\text { n.s. }\end{array}$ & $\begin{array}{l}\text { n.s. } \\
\text { n.s. } \\
\text { n.s. } \\
\text { n.s. }\end{array}$ & $\begin{array}{cc}0 & 120 \\
\text { n.d. } & \text { n.d. } \\
40 & 120 \\
\text { n.d. } & \text { n.d. }\end{array}$ & $\begin{array}{c}0 \\
\text { n.d. } \\
0 \\
\text { n.d. }\end{array}$ & $\begin{array}{c}0 \\
\text { n.d. } \\
0 \\
\text { n.d. }\end{array}$ & $\begin{array}{l}\text { n.s. } \\
\text { n.s. } \\
\text { n.s. } \\
\text { n.s. }\end{array}$ & $\begin{array}{l}\text { n.s. } \\
\text { n.s. } \\
\text { n.s. } \\
\text { n.s. }\end{array}$ \\
\hline & 3 & $\begin{array}{c}\text { HAS DSP } \\
\text { DSP TOXINS } \\
\text { HAS DSP } \\
\text { DSP TOXINS } \\
\text { HAS DSP } \\
\text { DSP TOXINS }\end{array}$ & $\begin{array}{c}686,560 \\
\text { n.d. } \\
1800 \\
192.5 \\
180,557 \\
1092\end{array}$ & $\begin{array}{c}20,200 \\
\text { n.d. } \\
8200 \\
\text { n.d. } \\
14,800 \\
188.2\end{array}$ & $\begin{array}{l}\text { n.s. } \\
\text { n.s. } \\
\text { n.s. } \\
\text { n.s. } \\
\text { n.s. } \\
\text { n.s. }\end{array}$ & $\begin{array}{l}\text { n.s. } \\
\text { n.s. } \\
\text { n.s. } \\
\text { n.s. } \\
\text { n.s. } \\
\text { n.s. }\end{array}$ & $\begin{array}{l}\text { n.s. } \\
\text { n.s. } \\
\text { n.s. } \\
\text { n.s. } \\
\text { n.s. } \\
\text { n.s. }\end{array}$ & $\begin{array}{c}2200 \\
135.7^{*} \\
1600 \\
\text { n.d. } \\
2400 \\
301.3\end{array}$ & $\begin{array}{c}60,000 \\
\text { n.d. } \\
20,200 \\
\text { n.d. } \\
19,200 \\
181.7\end{array}$ & $\begin{array}{l}\text { n.s. } \\
\text { n.s. } \\
\text { n.s. } \\
\text { n.s. } \\
\text { n.s. } \\
326.5\end{array}$ & $\begin{array}{l}\text { n.s. } \\
\text { n.s. } \\
\text { n.s. } \\
\text { n.s. } \\
\text { n.s. }\end{array}$ & $\begin{array}{l}\text { n.s. } \\
\text { n.s. } \\
\text { n.s. } \\
\text { n.s. } \\
\text { n.s. } \\
\text { n.s. }\end{array}$ & $\begin{array}{l}\text { n.s. } \\
\text { n.s. } \\
\text { n.s. } \\
\text { n.s. } \\
\text { n.s. } \\
\text { n..s. }\end{array}$ & $\begin{array}{l}\text { n.s. } \\
\text { n.s. } \\
\text { n.s. } \\
\text { n.s. } \\
\text { n.s. }\end{array}$ & $\begin{array}{l}\text { n.s. } \\
\text { n.s. } \\
\text { n.s. } \\
\text { n.s. } \\
\text { n.s. }\end{array}$ & $\begin{array}{c}17400 \\
\text { n.d. } \\
0 \\
\text { n.d. } \\
9400 \\
210.1\end{array}$ & $\begin{array}{l}1120 \\
\text { n.d. } \\
1240 \\
\text { n.d. } \\
7800 \\
\text { n.d. }\end{array}$ & $\begin{array}{l}\text { n.s. } \\
\text { n.s. } \\
\text { n.s. } \\
\text { n.s. } \\
\text { n.s. } \\
\text { n.s. }\end{array}$ & $\begin{array}{l}\text { n.s. } \\
\text { n.s. } \\
\text { n.s. } \\
\text { n.s. } \\
\text { n.s. } \\
\text { n.s. }\end{array}$ & $\begin{array}{l}\text { n.s. } \\
\text { n.s. } \\
\text { n.s. } \\
\text { n.s. } \\
\text { n.s. } \\
\text { n.s. }\end{array}$ & $\begin{array}{l}\text { n.s. } \\
\text { n.s. } \\
\text { n.s. } \\
\text { n.s. } \\
\text { n.s. } \\
\text { n.s. }\end{array}$ & $\begin{array}{l}\text { n.s. } \\
\text { n.s. } \\
\text { n.s. } \\
\text { n.s. } \\
\text { n.s. } \\
\text { n.s. }\end{array}$ & $\begin{array}{l}\text { n.s. } \\
\text { n.s. } \\
\text { n.s. } \\
\text { n.s. } \\
\text { n.s. } \\
\text { n.s. }\end{array}$ & $\begin{array}{l}18,2003000 \\
\text { n.d. n.d. } \\
10,800600 \\
\text { n.d. n.d. } \\
3600 \text {. } 1000 \\
\text { n.d. n.d. }\end{array}$ & $\begin{array}{l}\text { n.s. } \\
\text { n.s. } \\
\text { n.s. } \\
\text { n.s. } \\
\text { n.s. } \\
\text { n.s. }\end{array}$ & $\begin{array}{l}\text { n.s. } \\
\text { n.s. } \\
\text { n.s. } \\
\text { n.s. } \\
\text { n.s. } \\
\text { n.s. }\end{array}$ & $\begin{array}{l}\text { n.s. } \\
\text { n.s. } \\
\text { n.s. } \\
\text { n.s. } \\
\text { n.s. } \\
\text { n.s. }\end{array}$ & $\begin{array}{l}\text { n.s. } \\
\text { n.s. } \\
\text { n.s. } \\
\text { n.s. } \\
\text { n.s. } \\
\text { n.s. }\end{array}$ \\
\hline RO & 4 & $\begin{array}{c}\text { HAS DSP } \\
\text { DSP TOXINS } \\
\text { HAS DSP } \\
\text { DSP TOXINS }\end{array}$ & $\begin{array}{c}25,600 \\
\text { n.d. } \\
62,600 \\
\text { n.d. }\end{array}$ & $\begin{array}{l}\text { n.s. } \\
\text { n.s. } \\
\text { n.s. } \\
\text { n.s. }\end{array}$ & $\begin{array}{l}\text { n.s. } \\
\text { n.s. } \\
\text { n.s. } \\
\text { n.s. }\end{array}$ & $\begin{array}{l}\text { n.s. } \\
\text { n.s. } \\
\text { n.s. } \\
\text { n.s. }\end{array}$ & $\begin{array}{l}\text { n.S. } \\
\text { n.S. } \\
\text { n.S. } \\
\text { n.S. }\end{array}$ & $\begin{array}{c}13,200 \\
\text { n.r. } \\
14,000 \\
\text { n.d. }\end{array}$ & $\begin{array}{l}\text { n.s. } \\
\text { n.s. } \\
\text { n.s. } \\
\text { n.s. }\end{array}$ & $\begin{array}{l}\text { n.s. } \\
\text { n.s. } \\
\text { n.s. } \\
\text { n.s. }\end{array}$ & $\begin{array}{l}\text { n.s. } \\
\text { n.s. } \\
\text { n.s. } \\
\text { n.s. }\end{array}$ & $\begin{array}{l}\text { n.s. } \\
\text { n.s. } \\
\text { n.s. } \\
\text { n.s. }\end{array}$ & $\begin{array}{l}\text { n.s. } \\
\text { n.s. } \\
\text { n.s. } \\
\text { n.s. }\end{array}$ & $\begin{array}{l}\text { n.s. } \\
\text { n.s. } \\
\text { n.s. } \\
\text { n.s. }\end{array}$ & $\begin{array}{l}\text { n.s. } \\
\text { n.s. } \\
\text { n.s. } \\
\text { n.s. }\end{array}$ & $\begin{array}{c}0 \\
\text { n.r. } \\
0 \\
\text { n.d. }\end{array}$ & $\begin{array}{l}1560 \\
184 \\
1680 \\
205\end{array}$ & $\begin{array}{l}\text { n.s. } \\
\text { n.s. } \\
\text { n.s. } \\
\text { n.s. }\end{array}$ & $\begin{array}{l}\text { n.s. } \\
\text { n.s. } \\
\text { n.s. } \\
\text { n.s. }\end{array}$ & $\begin{array}{l}\text { n.s. } \\
\text { n.s. } \\
\text { n.s. } \\
\text { n.s. }\end{array}$ & $\begin{array}{l}\text { n.s. } \\
\text { n.s. } \\
\text { n.s. } \\
\text { n.s. }\end{array}$ & $\begin{array}{l}\text { n.s. } \\
\text { n.s. } \\
\text { n.s. } \\
\text { n.s. }\end{array}$ & $\begin{array}{l}\text { n. } s \\
\text { n. } s \\
\text { n. } s \\
\text { n. } s\end{array}$ & $\begin{array}{l}22,60023,800 \\
\text { n.d. n.d. } \\
19,40012,400 \\
\text { n.d. n.d. }\end{array}$ & $\begin{array}{r}0 \text { n.s. } \\
\text { n.s. } \\
0 \text { n.s. } \\
\text { n.s. }\end{array}$ & $\begin{array}{l}\text { n.s. } \\
\text { n.s. } \\
\text { n.s. } \\
\text { n.s. }\end{array}$ & $\begin{array}{l}\text { n. } s \\
\text { n. } s \\
\text { n. } s \\
\text { n.s }\end{array}$ & $\begin{array}{l}\text { s n.s. } \\
\text { s n.s. } \\
\text { s n.s. } \\
\text { s n.s. }\end{array}$ \\
\hline
\end{tabular}

COL, Colostrai; FER, Feraxi; SGN, San Giovanni; SGL, Santa Gilla; TOR, Tortolì; ORO, Orosei; HAS, harmful algal species; DSP, diarrheic shellfish poisoning; DSP TOXINS, okadaic acid group and total derivates quantification; HAS DSP, toxic harmful algal species densities; n.d., not detectable below of limit of quantification; n.s., not sampled; n.r., not retrieved. The progressive sample sequencing monthly is indicated with Roman numerals. In italics are the toxins values that exceed the legal limit. *Toxin presence not overcoming the legal limit. 
They were the most important algae observed among all the potentially toxic HAS observed, regarding cell abundance and distribution. Nonetheless, ASP toxicity in mussels has not been detected, apart from one case (in March in SGL in the station 2), in which the value, however, remained within legal limits allowed. Concurrently, Pseudo-nitzschia spp. concentration was about $11 \times 10^{3} \mathrm{cel} / \mathrm{L}$.

\section{Discussion}

The occurrence of potentially toxic HAS and the accumulation of algal toxins are critical problems which are growing worldwide in bivalve aquaculture (Economou et al., 2007) which is growing worldwide. The three most important kinds of poisoning shellfish are PSP, DSP and ASP. About the $70 \%$ of a list of marine species identified as toxin-producing microalgae is represented by Dinoflagellates (Garcia Camacho et al., 2007) and the phycotoxin they produce is among the most poisoning biotoxin known (Van Egmond, 2004). The aim of Sardinian regional monitoring of harmful phytoplankton and biotoxins in mussel farm areas is to minimize the risk of intoxication. This permits to give an early warning and, if needed, an immediate closing of the shellfish farm, preventing human illnesses (van der FelsKlerx et al., 2012).

During this study, DSP toxins were detected in several shellfish samples from Cagliari and East areas. Our results seems to suggest that there is no possible link between the toxins presence in shellfish, and the occurrence of toxic species, although a slight relation between DSP toxins and Dinophysis species could be supported. Alexandrium species were present in Oristano area only, just in seven of the total samples, in which PSP toxins were reported once (in CSM) without even exceed the alert safety limit. In this case, the first biological tests were positive, so there was a precautionary closing of the shellfish farm. Subsequent analysis resulted negative and this allowed the immediate reopening of the area. In Sardinia, PSP toxicity was first recorded in late spring 2002 in Olbia area (Virgilio et al., 2003) with the presence of $A$. minutum and $A$. catenella, while in November 2006 the mussels showed PSP contamination in GOR. PSP events were also documented in the following years in both areas (Virgilio et al., 2010; Lorenzoni et al., 2011). From January 2012 to present days, no new PSP positive events were detected in Sardinian water. The incidence of PSP toxicity in Oristano area was documented only in Autumn (October-November), whereas in Olbia it was documented in Spring or Winter, so it turned out to be a site-dependent seasonality (Lugliè et al., 2011; Lorenzoni et $a l ., 2013)$. In this study, positivity presence has been reported in Oristano area in March, so it seems that Alexandrium species may have expanded their presence regarding time. The results of this study are in agreement with statistical previous long-term studies carried out in Sardinia in which the occurrence of $A$. minutum significantly decreased in Olbia area (Bazzoni et al., 2015), although it is important to notice that in the past most PSP-positive events that took place in Olbia area, caused economic losses due to the shellfish harvest quitting (Lugliè et al., 2011; Lorenzoni et al., 2013). These facts make clear how difficult it is to investigate any possible correlation between PSP phytoplankton and PSP toxins, although the presence of PSP toxin coincides with the highest Alexandrium abundance detected during the study (800 cell/L) (Figure 2). Anyway, in some cases there was Alexandrium presence but the toxin was absent.

Several potentially causative agents of DSP were found in Sardinia (Dinophysis and Prorocentrum species). Despite the presence of many DSP phytoplankton species, only $D$. acuminata seems to have contributed to the positivity in shellfish during the period of this study because it appears in higher concentrations during DSP events. The interpretation of the relations between DSP toxins and Dinophysis species results quite difficult, also because our results are collected in a short temporal scale. Anyway, in many cases mussels toxicity and Dinophysis presence have not occurred simultaneously, namely a decrease in

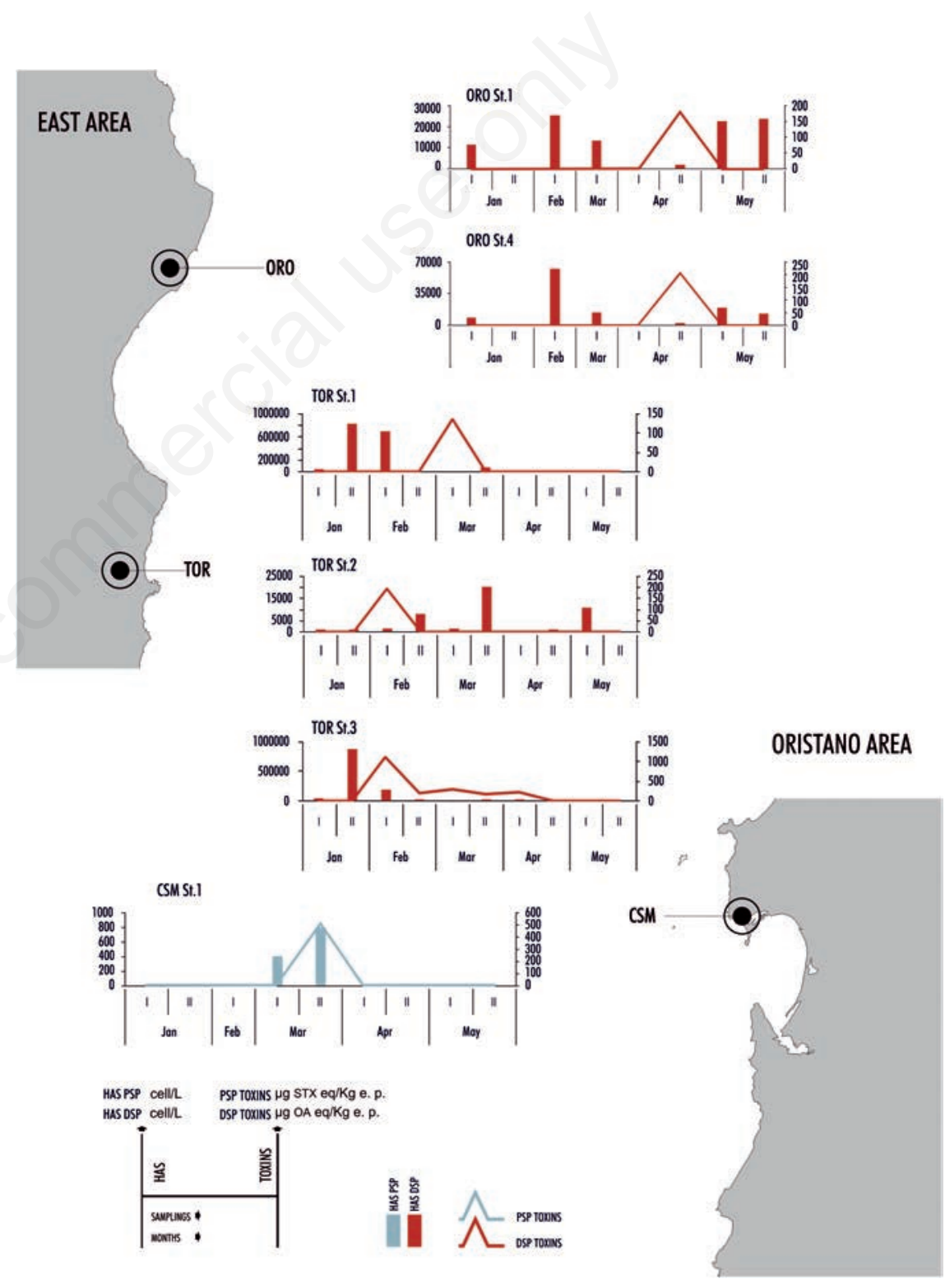

Figure 2. Evolution of potentially toxic harmful algal species densities and toxins concentration in the East and Oristano areas from January to May 2015. 
microalga densities occurred before the peak of DSP toxins concentration. This was particularly evident in FER, TOR and ORO (Figure 2). SGL can be considered too, taking into account that DSP phytoplankton was present in November and December 2014 (data not published). These dynamics may depend on a period of toxin accumulation, which is then released when the algal abundance decrease. The accumulation times were different in the different areas. In TOR and ORO they were shorter (about two weeks) than in SGL (about a month). In the first five months of 2015 , there have been several DSP positive events (23), which occurred more commonly than PSP cases (only one). This was the exact opposite of what happened in the past when DSP occurred less frequently compared to PSP (the only reports there were in 2002 and 2003 in the SGL and FER) (Lugliè et al.).
Pseudo-nitzschia was the most important potentially toxic genus due the high abundance observed in all sites in different occasions. However, ASP toxicity in mussels has been detected only once, with a value markedly below the regulatory level of DA $(20 \mathrm{mg} \mathrm{DA} / \mathrm{kg}$ e.p.). This finding supports past years results (Bazzoni et al., 2015), in which there was not any relation between the very high abundance of Pseudo-nitzschia species, and the occurrence of toxicity events. The massive presence of the genus reported is probably related to the fact that the toxic and no-toxic Pseudonitzschia species have not been distinguished and counted together.

The data obtained in this study show a quite unexpected increase in the toxicity of mussels in Sardinia. In fact, in 2015 many DSP toxicity episodes have occurred, after several years of their absence. Despite this, DSP phytoplank-
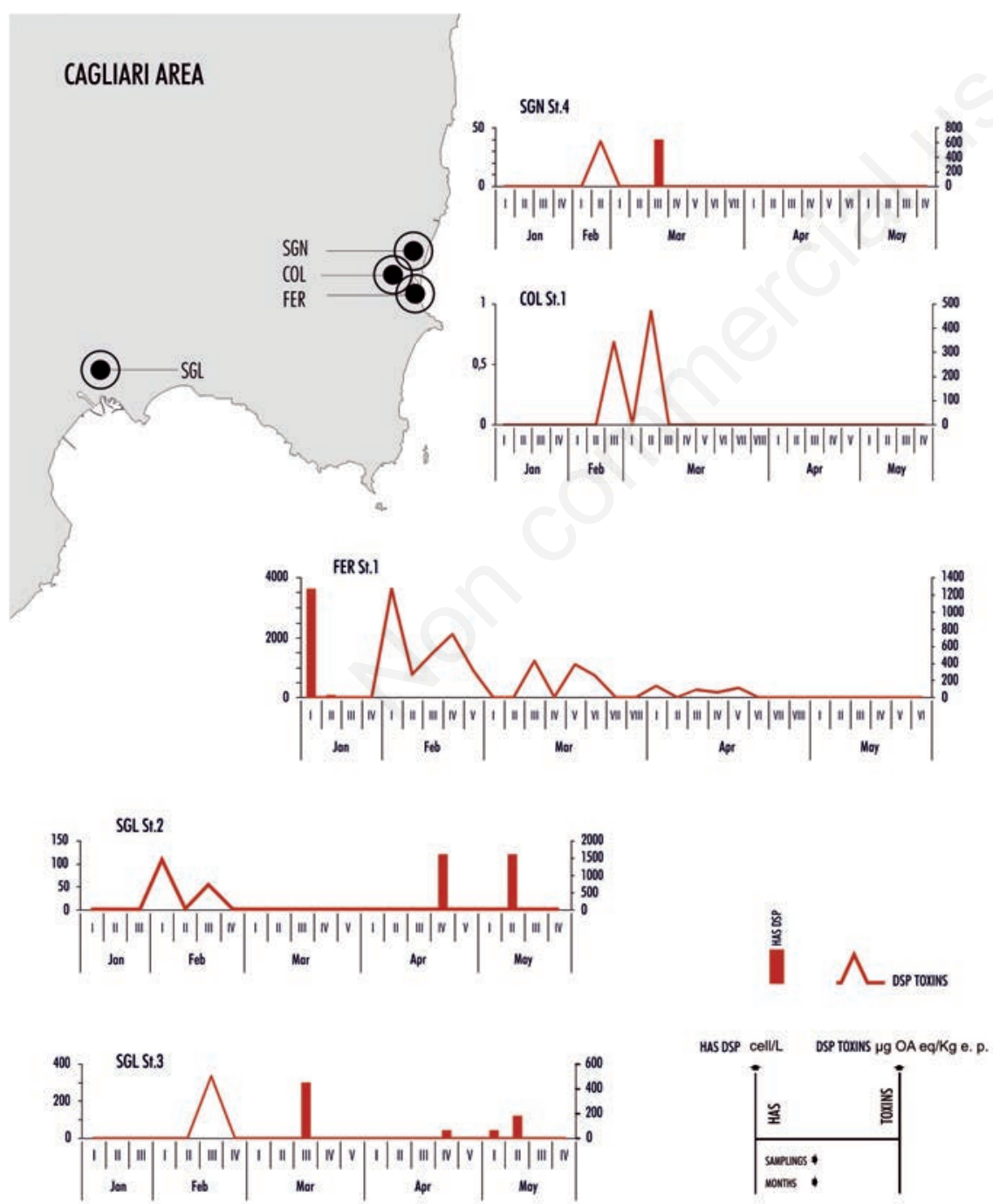

Figure 3. Evolution of potentially toxic harmful algal species densities and toxins concentration in Cagliari area from January to May 2015.

ton has been constantly present. Consequently, algal toxins and related human poisoning postbivalve consummation are a real and continuing problem. Moreover, although late autumnwinter and spring seem to be the most dangerous periods of toxic events, a specific seasonality does not properly exist, and the positive samples can be detected during all months.

\section{Conclusions}

Many efforts have to be made to better understand the relation between shellfish toxicity and potentially toxic HAS densities. In particularly, Dinoflagellates may sometimes have densities as low as one to several hundred cell/L leading to the accumulation of toxin above regulatory level, or the total toxin content may increase at the end of a bloom when Dinoflagellates abundance decline, thus the risk of shellfish toxicity can persist even when cell abundance is lower. These considerations represent a challenge in the realisation of monitoring programmes. Future monitoring designs should include all factors (biologic, physical, physiological) which can help to make clear the relation between potentially toxic HAS and toxic phenomena in the bivalve molluscs.

\section{References}

AESAN, 2015. EU-harmonised standard operating procedure for determination of lipophilic marine biotoxins in molluscs by LCMS/MS. Version 5. European Union Reference Laboratory for Marine Biotoxins, Agencia Española de Consumo, Seguridad Alimentaria y Nutrición, Vigo, Spain.

AOAC, 2005. Officinal methods of analysis. 18th ed. Association of Official Analytical Chemists ed., Gaithersburg, MD, USA.

Bazzoni AM, Caddeo T, Pulina S, Padedda BM, Satta CT, Sechi N, Lugliè A, 2015. Spatial distribution and multiannual trends of potentially toxic microalgae in shellfish farms along the Sardinian coast (NW Mediterranean Sea). Environ Monit Assess 187:86.

Economou V, Papadopoulou C, Brett M, Kansouzidou A, Charalabopoulos K, Filioussis G, Seferiadis K, 2007. Diarrhetic shellfish poisoning due to toxic mussel consumption: the first record outbreak in Greece. Food Addit Contam 24:297-305.

European Commission, 2004. Regulation of the European Parliament and of the Council of 29 April 2004 laying down speci- 
fic hygiene rules for on the hygiene of foodstuffs, 853/2004/EC. In: Official Journal, L 139, 30/04/2004.

European Commission, 2011. Regulation of 10 January 2011 amending Regulation (EC) No 2074/2005 as regards recognised testing methods for detecting marine biotoxins in live bivalve molluscs, 15/2011/CE. In: Official Journal, L 6, 10/01/2011.

Garcia Camacho F, Gallardo Rodríguez J, Sánchez Mirón A, Cerón García MC, Belarbi EH, Chisti Y, Molina Grima E, 2007. Biotechnological significance of toxic marine dinoflagellates. Biotech Adv 25:176-94.

Hallegraeff GM, 2010. Ocean climate change, phytoplankton community response, and harmful algal blooms: a formidable predictive challenge. J Phycol 46:220-35.

Lorenzoni G, Arras I, Sanna G, Delogu P, Mudadu A, Piras A, Mura A, Marongiu E, Virgilio S, 2013. Paralytic shellfish poison algal biotoxins: Sardinia report 2002-2011 and non-compliance management. Ital $\mathrm{J}$ Food Safety 2:104-8.

Lorenzoni G, Tedde T, Terrosu G, Fattaccio C, Salza S, Arras I, Sanna G, Canu A, Uda MT, Marongiu E, Virgilio S, 2011. [La gestione delle non conformità da biotossine algali del tipo P.S.P. (Paralytic Shellfish Poison) in mitili allevati e commercializzati nella regione Sardegna]. [Article in Italian]. Ital J Food Safety 2:95-6.

Lugliè A, Satta CT, Pulina S, Bazzoni AM, Padedda BM, Sechi N, 2011. Harmful algal blooms in Sardinia. Biol Mar Med 18:2-9.

Mazzette R, Virgilio S, Piras A, Tempesta A, Serra S, Sferlazzo G, Pisano M, Mureddu A, Meloni D, 2010. [Valutazione dell'efficacia dei sistemi di depurazione nei confronti di E. coli, Salmonella spp. e Vibrio spp. in mitili allevati nel Golfo di Olbia]. [Article in Italian]. Il Pesce 5:165-70.

Regione Sardegna, 2014. [Piano regionale per la vigilanza ed il controllo sanitario della produzione e commercializzazione dei molluschi bivalvi vivi e per il monitoraggio periodico delle zone di produzione e di stabulazione di molluschi bivalvi vivi, anno 2014]. [Regulation in Italian]. Regione Sardegna ed., Sassari, Italy.

Sannio A, Lugliè A, Sechi N, 1997. Potentially toxic dinoflagellates in Sardinia. Plant Biosyst 131:73-8.

Taleb H, Vale P, Blaghen M, 2003. Spatial and temporal evolution of PSP toxins along the Atlantic shore of Morocco. Toxicon 41:199205.
Utermöhl H, 1958. [Zur vervollkommung der quantitativen phytolankton-methodik]. [Article in German]. Verh Int Verein Theor Angew Limnol 9:1-39.

van der Fels-Klerx HJ, Adamse P, Goedhart PW, Poelman M, Pol-Hofstad IE, van Egmond H, Gerssen A, 2012. Monitoring phytoplankton and marine biotoxins in production waters of Netherlands: results after one decade. Food Addit Contam 29:1616-29.

Van Egmond HP, 2004. Natural toxins: risks, regulation and the analytical situation in Europe. Anal Bioanal Chem 378:1152-60.

Virgilio S, Marongiu E, Pisanu M, Mancuso R, Piras A, Virdis F, Saba A, Lorenzoni G, Rosa MN, Carusillo F, Arras I, Sias S, Poletti R, 2003. [Riscontro di biotossine algali del tipo P.S.P. (Paralitic Shellfish Poisoning) in mitili allevati nel Golfo di Olbia, Sardegna]. [Article in Italian]. In: Proceedings of the XIII National A.I.V.I. Congress, Pescara, 6-8 June, pp. 209-13.

Virgilio S, Lorenzoni G, Tedde T, Terrosu G, Campus G, Rosa MN, Mura A, Delogu P, Piras M, 2010. Presence of P.S.P. toxins (Paralytic Shellfish Poison) in mussels of Sardinia and non conformity management. Ital J Food Safety 8:71. 